Acta Crystallographica Section F

Structural Biology

and Crystallization

Communications

ISSN 1744-3091

\section{Qiao-Ming Hou, ${ }^{\mathrm{a}, \mathrm{b}}$ Xiang Liu, Erik Brostromer, ${ }^{\mathrm{b}}$ Lan-Fen $\mathrm{Li}^{\mathrm{b}}$ and Xiao-Dong $\mathrm{Su}^{\mathrm{a}, \mathrm{b} *}$}

aPeking University Shenzhen Graduate School, Shenzhen 518055, People's Republic of China, and ${ }^{\mathbf{b}}$ The National Laboratory of Protein Engineering and Plant Genetic Engineering, College of Life Sciences, Peking University, Beijing 100871, People's Republic of China

Correspondence e-mail: xdsu@pku.edu.cn

Received 29 July 2009

Accepted 28 October 2009

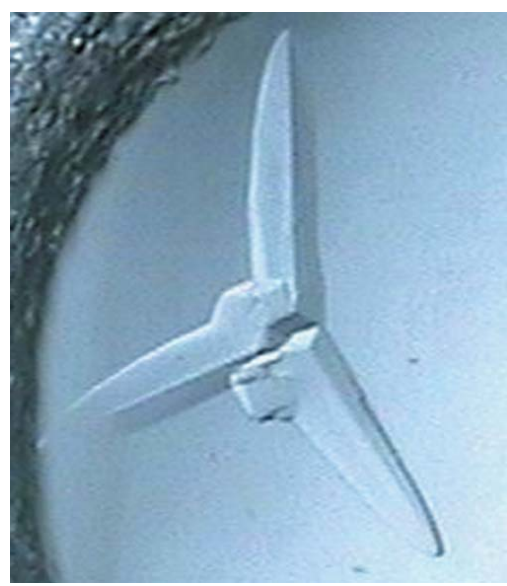

(C) 2009 International Union of Crystallography All rights reserved

\section{Preliminary crystallographic studies of purine nucleoside phosphorylase from the cariogenic pathogen Streptococcus mutans}

The punA gene of the cariogenic pathogen Streptococcus mutans encodes purine nucleoside phosphorylase (PNP), which is a pivotal enzyme in the nucleotidesalvage pathway, catalyzing the phosphorolysis of purine nucleosides to generate purine bases and $\alpha$-ribose 1-phosphate. In the present work, the PNP protein was expressed in Escherichia coli strain BL21 (DE3) in a soluble form at a high level. After purification of the PNP enzyme, the protein was crystallized using the sitting-drop vapour-diffusion technique; the crystals diffracted to $1.6 \AA$ resolution at best. The crystals belonged to space group $H 3$, with unit-cell parameters $a=b=113.0, c=60.1 \AA$.

\section{Introduction}

Purine nucleoside phosphorylase (PNP; EC 2.4.2.1) is a key enzyme in the nucleotide-salvage pathway, catalyzing reversible cleavage of the $\mathrm{N}$-glycosidic bond of purine nucleosides to generate free purine bases and $\alpha$-ribose 1-phosphate. The nucleotide-salvage pathway enables the organisms to synthesize new nucleotides using bases and $\alpha$-ribose 1-phosphate metabolized from ribonucleosides and deoxyribonucleosides, which is an energy-saving pathway compared with the de novo nucleotide-synthesis pathway (Bzowska et al., 2000). According to the oligomeric state and substrate specificity of the protein, PNPs can be classified into two groups. Trimeric forms such as human PNP, which are found mainly in mammals and some bacteria, are specific for 6-oxopurine nucleoside substrates (inosine and guanosine). Hexameric forms such as Escherichia coli PNP, which occur in most bacteria, accept both 6-oxopurine nucleosides and 6-aminopurine nucleosides (adenosine) as substrates (Bzowska et al., 2000). The crystal structures of several trimeric and hexameric PNPs have been reported (Ealick et al., 1990; Bzowska et al., 1995; Mao et al., 1997; Koellner et al., 1998; Tebbe et al., 1999). Despite the fact that the amino-acid sequences, substrate specificities and oligomeric states differ between the two kinds of PNPs, their subunits exhibit similar structural features (Pugmire \& Ealick, 2002). Each monomer displays an $\alpha / \beta$-fold. The core structure consists of a mixed $\beta$-sheet which forms a distorted $\beta$-barrel surrounded by several $\alpha$-helices. The $\alpha$-helices and $\beta$-strands are linked by extended loops.

Several aspects of PNP have led to its proposal as a potential drug target. For instance, human PNP inhibitors can be used to treat T-cell leukaemia and lymphomas (Kazmers et al., 1981) and can activate some antitumour and antiviral nucleoside drugs in vivo (Bennett $e t$ al., 1993). Immucillin-H is an example of a picomolar inhibitor that was designed based on the PNP transition-state structure (Kicska et al., 2001). Moreover, PNP from E. coli is a candidate in tumour gene therapy owing to the differences in substrate specificity between the bacterial and mammalian PNPs (Sorscher et al., 1994; Hughes et al., 1995).

Streptococcus mutans has been implicated as a principal causative agent of human dental caries (Loesche, 1986) and infective endocarditis (Ullman et al., 1988). S. mutans PNP, a 269-residue protein with a molecular weight of $28.8 \mathrm{kDa}$, is encoded by the punA gene and shows $46 \%$ sequence identity to human and bovine PNP. Studies 
of the crystal structure of $S$. mutans PNP should supply helpful clues for the therapy of dental caries owing to its critical role in purine nucleoside metabolism.

\section{Experimental procedures}

\subsection{Cloning, expression and purification}

The punA gene (Gene ID 1028527) was cloned from S. mutans genomic DNA by polymerase chain reaction (PCR) using primers $5^{\prime}$ CGCGGATCCATGTCATTACTTAAAAAAATTTAT- ${ }^{\prime}$ and 5'-CCGCTCGAGTTATAATTCTACTAAAATAGCTTT-3', which contain BamHI and XhoI restriction sites, respectively. The target gene was inserted into a pET28a expression vector (Novagen) using the conventional cloning method. An N-terminal $\mathrm{His}_{6}$ tag with sequence MGSSHHHHHHSSGLVPRGSHMASMTGGQQMGRGS was fused to the gene product. The final constructed vector was verified by DNA sequencing.

The constructed plasmid was transformed into E. coli strain BL21 (DE3) cells (Invitrogen) for protein expression. The transformants were grown in $20 \mathrm{ml}$ Luria-Bertani (LB) medium containing kanamycin $\left(50 \mu \mathrm{g} \mathrm{ml}^{-1}\right)$ at $310 \mathrm{~K}$ overnight. The overnight culture was then added to 11 of fresh $\mathrm{LB}$ medium for growth. When an $\mathrm{OD}_{600}$ of 0.6 was reached, isopropyl $\beta$-D-1-thiogalactopyranoside was added to the cell culture to a final concentration of $0.5 \mathrm{~m} M$ for induction. After incubation for a further $4 \mathrm{~h}$ at $310 \mathrm{~K}$, cells were harvested by $15 \mathrm{~min}$ centrifugation at $5100 \mathrm{~g}$ and $277 \mathrm{~K}$.

The cell pellet was then resuspended in $20 \mathrm{ml}$ lysis buffer $(20 \mathrm{mM}$ Tris- $\mathrm{HCl}, 500 \mathrm{~m} M \mathrm{NaCl} \mathrm{pH} 7.5$ ) and disrupted by sonication on ice. After $40 \mathrm{~min}$ centrifugation at $39000 \mathrm{~g}$ and $277 \mathrm{~K}$, the supernatant was filtered using a $0.22 \mu \mathrm{m}$ filter before purification of the target protein by two-step chromatography. The supernatant was first applied onto a $5 \mathrm{ml} \mathrm{Ni}{ }^{2+}$-chelating affinity column (HiTrap, GE Healthcare, USA) previously equilibrated with lysis buffer. The column was first washed with lysis buffer to remove unbound proteins and was then washed with lysis buffer containing $50 \mathrm{~m} M$ imidazole to remove nonspecifically bound proteins. The target protein was finally eluted using a linear gradient of imidazole from 50 to $500 \mathrm{~m} M$ in lysis buffer. Fractions containing the target protein were collected and

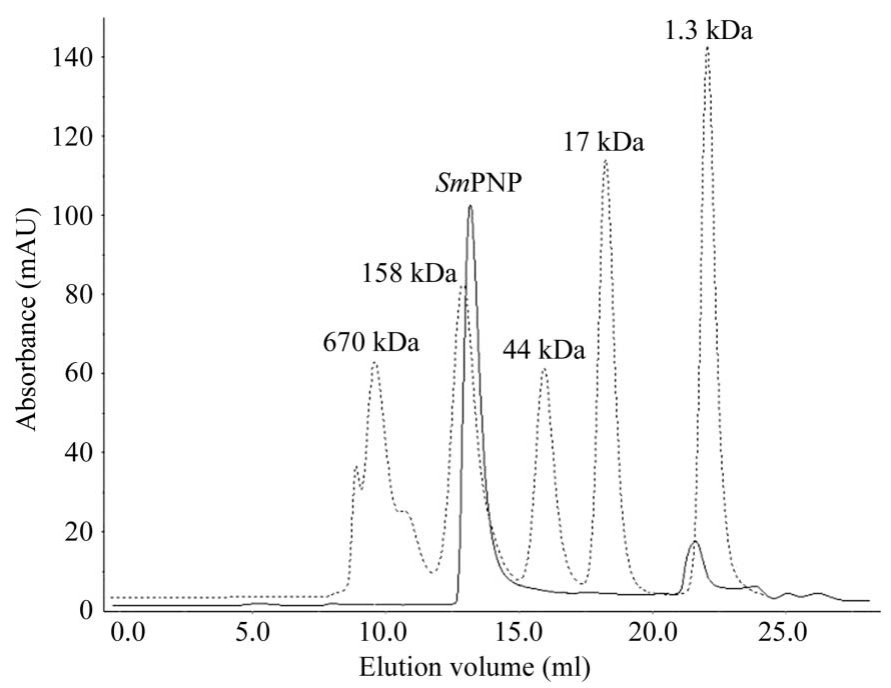

Figure 1

Chromatogram elution profile of $S$. mutans PNP ( $S m \mathrm{PNP})$ on a Superdex 75 gelfiltration column. The absorption curves of gel-filtration standard proteins (BioRad Laboratories, USA) and SmPNP are shown as dashed and solid lines, respectively. concentrated to about $1.5 \mathrm{ml}$ using an ultrafiltration device (Ultra-15, $10 \mathrm{kDa}$ cutoff, Millipore, USA) for the next purification step. The target protein was further purified using a $120 \mathrm{ml}$ Superdex 75 gelfiltration column (HiLoad, GE Healthcare, USA) pre-equilibrated with a buffer solution containing $20 \mathrm{~m} M$ Tris- $\mathrm{HCl}, 200 \mathrm{mM} \mathrm{NaCl}$ $\mathrm{pH}$ 7.5. The purity of the target protein was checked by SDS-PAGE analysis.

\subsection{Crystallization}

The purified protein from the gel filtration was concentrated to about $15 \mathrm{mg} \mathrm{ml}^{-1}$ for crystallization trials. The protein concentration was measured using a Bio-Rad protein-assay kit (Bio-Rad Laboratories, USA) with bovine serum album as a standard. Crystallization screening was performed at $293 \mathrm{~K}$ using the sitting-drop vapourdiffusion method with an XtalQuest $48^{2}$ crystallization plate (XtalQuest, Beijing, People's Republic of China). Crystal Screen, Crystal Screen 2 and Index Screen kits (Hampton Research, USA) were used for initial screening. $1 \mu \mathrm{l}$ protein solution (containing $20 \mathrm{mM}$ Tris$\mathrm{HCl}, 200 \mathrm{~m} M \mathrm{NaCl} \mathrm{pH} 7.5$ ) was mixed with $1 \mu \mathrm{l}$ reservoir solution and equilibrated against $100 \mu \mathrm{l}$ reservoir solution in each condition.

\subsection{Data collection and processing}

X-ray diffraction data were collected using a MAR Mosaic 225 CCD detector on beamline I911-3, MAX-lab, Lund University, Sweden. The crystal-to-detector distance was set to $330 \mathrm{~mm}$ and the wavelength was $0.979 \AA$ A. The crystal was flash-cooled without any cryoprotectant and was maintained at $100 \mathrm{~K}$ during data collection using a cold nitrogen stream. A total of 150 frames were collected with $1^{\circ} \varphi$ oscillation per frame. Data were processed using the $X D S$ software suite (Kabsch, 1993).

\section{Results}

The $S$. mutans punA gene was verified to be correctly cloned into the pET28a expression vector by DNA sequencing. The $S$. mutans PNP protein was expressed in a soluble form at a high level. The typical yield of the protein was about $30 \mathrm{mg}$ pure protein per litre of cell culture. The results of SDS-PAGE showed that the molecular mass of the fusion target protein was $\sim 33 \mathrm{kDa}$, which is consistent with the

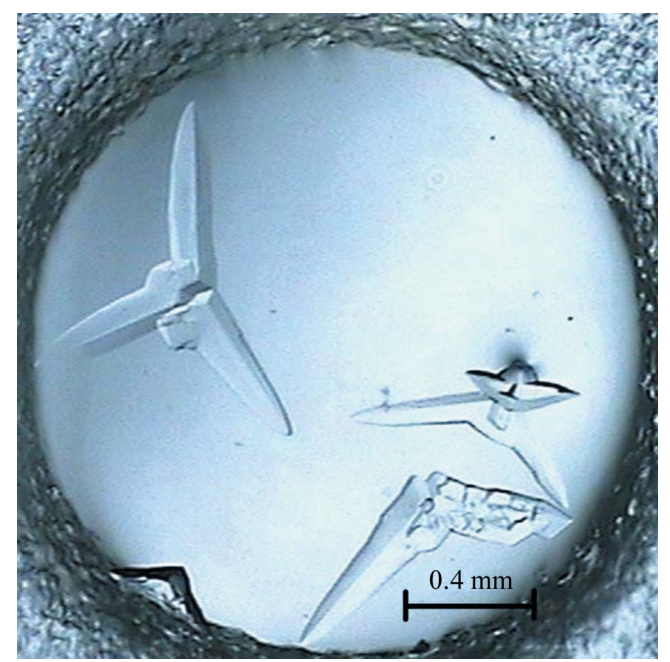

Figure 2

Crystals of $S$. mutans PNP protein grown by the sitting-drop vapour-diffusion method using a reservoir solution containing 25\%(w/v) PEG 3350, $0.15 \mathrm{M}$ ammonium sulfate and $0.1 M$ bis-tris $\mathrm{pH} 6.3$. 
Table 1

Data-collection statistics for S. mutans PNP.

Values in parentheses are for the highest resolution shell.

\begin{tabular}{ll}
\hline Resolution $(\AA)$ & $18.8-2.0(2.1-2.0)$ \\
Completeness $(\%)$ & $90.2(100.0)$ \\
$R_{\text {merge }}^{\dagger}(\%)$ & $4.0(8.1)$ \\
Mean $I / \sigma(I)$ & $29.6(16.8)$ \\
Space group & $H 3$ \\
Unit-cell parameters $(\AA)$ & $a=b=113.0, c=60.1$ \\
No. of observed reflections & $87487(13240)$ \\
No. of unique reflections & $17443(2603)$ \\
Molecules per ASU & 1 \\
$V_{\mathrm{M}} \ddagger\left(\AA^{3} \mathrm{Da}^{-1}\right)$ & 2.6 \\
Solvent content $(\%)$ & 53
\end{tabular}

$\dagger R_{\text {merge }}=\sum_{h k l} \sum_{i}\left|I_{i}(h k l)-\langle I(h k l)\rangle\right| / \sum_{h k l} \sum_{i} I_{i}(h k l)$, where $I_{i}(h k l)$ is the $i$ th observation of reflection $h k l$ and $\langle I(h k l)\rangle$ is the mean intensity of all observations of reflection hkl. \$ Matthews (1968)

theoretical molecular mass of the PNP protein plus a hexahistidyl tag of $4.0 \mathrm{kDa}$. The $S$. mutans PNP protein was found to exist as a trimer in solution according to the gel-filtration chromatography profile (Fig. 1). In the initial crystallization screening, microcrystals appeared under several conditions in one week. Optimization of crystallization conditions was applied based on the condition $25 \%(w / v)$ polyethylene glycol (PEG) 3350, 0.2 $M$ ammonium sulfate and $0.1 M$ bistris pH 5.5 (Hampton Index Screen condition No. 66). Crystals suitable for X-ray diffraction were obtained from an optimized condition containing 25\%(w/v) PEG 3350, $0.15 \mathrm{M}$ ammonium sulfate and $0.1 M$ bis-tris $\mathrm{pH} 6.3$ (Fig. 2). The best crystal diffracted to maximum resolution of $1.6 \AA$. The data were finally collected and processed to $2.0 \AA$ resolution as the overall completeness of the data was a little low when processing at higher resolution. The phases of the structure were determined by the molecular-replacement method with the MOLREP program (Vagin \& Teplyakov, 1997) in the CCP4 suite (Collaborative Computational Project, Number 4, 1994) using the structure of bovine PNP (PDB code $1 \mathrm{fxu} ; 46 \%$ sequence identity to $S$. mutans PNP; Mao et al., 1998) as a search model. After the first round of rigid-body refinement with REFMAC (Murshudov et al., 1997 ), the $R_{\text {cryst }}$ and $R_{\text {free }}$ values were $35.8 \%$ and $39.8 \%$, respectively. Data-collection statistics are listed in Table 1. Refinement and analysis of the structure are in progress.
This work was supported by a grant from the National Natural Science Foundation of China (30530190). We gratefully acknowledge MAX-lab (Lund University, Sweden) for the synchrotron beam time.

\section{References}

Bennett, L. L. Jr, Allan, P. W., Noker, P. E., Rose, L. M., Niwas, S., Montgomery, J. A. \& Erion, M. D. (1993). J. Pharmacol. Exp. Ther. 266, 707-714.

Bzowska, A., Kulikowska, E. \& Shugar, D. (2000). Pharmacol. Ther. 88, 349-425.

Bzowska, A., Luić, M., Schröder, W., Shugar, D., Saenger, W. \& Koellner, G. (1995). FEBS Lett. 367, 214-218.

Collaborative Computational Project, Number 4 (1994). Acta Cryst. D50, 760-763.

Ealick, S. E., Rule, S. A., Carter, D. C., Greenhough, T. J., Babu, Y. S., Cook, W. J., Habash, J., Helliwell, J. R., Stoeckler, J. D., Parks, R. E. Jr, Chen, S. \& Bugg, C. E. (1990). J. Biol. Chem. 265, 1812-1820.

Hughes, B. W., Wells, A. H., Bebok, Z., Gadi, V. K., Garver, R. I. Jr, Parker, W. B. \& Sorscher, E. J. (1995). Cancer Res. 55, 3339-3345.

Kabsch, W. (1993). J. Appl. Cryst. 26, 795-800.

Kazmers, I. S., Mitchell, B. S., Dadonna, P. E., Wotring, L. L., Townsend, L. B. \& Kelley, W. N. (1981). Science, 214, 1137-1139.

Kicska, G. A., Long, L., Hörig, H., Fairchild, C., Tyler, P. C., Furneaux, R. H., Schramm, V. L. \& Kaufman, H. L. (2001). Proc. Natl. Acad. Sci. USA, 98, 4593-4598.

Koellner, G., Luić, M., Shugar, D., Saenger, W. \& Bzowska, A. (1998). J. Mol. Biol. 280, 153-166.

Loesche, W. J. (1986). Microbiol. Rev. 50, 353-380.

Mao, C., Cook, W. J., Zhou, M., Federov, A. A., Almo, S. C. \& Ealick, S. E. (1998). Biochemistry, 37, 7135-7146.

Mao, C., Cook, W. J., Zhou, M., Koszalka, G. W., Krenitsky, T. A. \& Ealick, S. E. (1997). Structure, 5, 1373-1383.

Matthews, B. W. (1968). J. Mol. Biol. 33, 491-497.

Murshudov, G. N., Vagin, A. A. \& Dodson, E. J. (1997). Acta Cryst. D53, 240-255.

Pugmire, M. J. \& Ealick, S. E. (2002). Biochem. J. 361, 1-25.

Sorscher, E. J., Peng, S., Bebok, Z., Allan, P. W., Bennett, L. L. Jr \& Parker, W. B. (1994). Gene Ther. 1, 233-238.

Tebbe, J., Bzowska, A., Wielgus-Kutrowska, B., Schröder, W., Kazimierczuk, Z., Shugar, D., Saenger, W. \& Koellner, G. (1999). J. Mol. Biol. 294, 12391255.

Ullman, R. F., Miller, S. J., Strampfer, M. J. \& Cunha, B. A. (1988). Heart Lung, 17, 209-212.

Vagin, A. \& Teplyakov, A. (1997). J. Appl. Cryst. 30, 1022-1025. 